\title{
Statistics of reactive ensembles on regular surfaces: application to benzene from acetylene on $\operatorname{Pd}(111)$
}

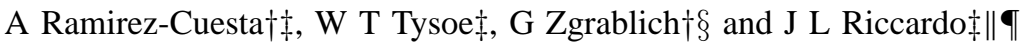 \\ $\dagger$ Departamento de Fisica (CONICET), Universidad Nacional de San Luis, Chacabuco y \\ Pedernera, (5700) San Luis, San Luis, Argentina \\ $\ddagger$ Department of Chemistry and Laboratory for Surface Studies, University of Wisconsin- \\ Milwaukee, Milwaukee, WI 53211, USA \\ $\S$ Centro Regional de Estudios Avanzados, CREA, CC 200, (5700) San Luis, Argentina \\ \| Department of Chemistry, 152 Davey Laboratory, The Pennsylvania State University, \\ University Park, PA 16802, USA
}

Received 18 July 1995, in final form 10 October 1995

\begin{abstract}
A simple model is proposed for calculating the reaction yield of a surface reaction of molecules chemisorbed on regular surfaces. An application to benzene from acetylene tricyclization on $\operatorname{Pd}(111)$ is performed. The analysis relies on random adsorption of acetylene on the clean crystal surface. The benzene yield is calculated in terms of the acetylene coverage. Analytical results are compared with computer simulation ones and experimental data. The predicted value for the maximum reaction efficiency as well as the behaviour as a function of the acetylene coverage are in fair agreement with experimental measurements.
\end{abstract}

\section{Introduction}

A number of processes in physics, chemistry and biology can be properly described by the random-sequential-adsorption (RSA) model on a lattice [1]. In particular RSA modelling has been successfully applied for many years to analytically calculating jamming (saturation) coverages of adsorbates on well-defined metal surfaces [2]. The random-filling model has also been used to study cyclization reaction where adjacent groups link [3], as well as to describe the dissociative adsorption of oxygen on $\mathrm{Ni}(100)$ [4] and $\operatorname{Pd}(100)[5,6]$. Ziff et al have introduced a diffusionless RSA + reaction model (ZGB) to study the monomer-dimer reaction [7]. Several surface reaction models have been modelled as RSA processes [8]. A very rich structure of the reacting phase, including unusual spatial correlations between the reactant molecules and nontrivial jamming coverages, is a very general feature that emerges from all of these systems.

In catalysis and surface reactions, one of the roles of the catalyst may be to correctly orient adsorbate molecules to allow further reaction to produce the final product. In some cases, this may lead to a strong surface structure sensitivity in the reaction. A good example of this 'template' effect is the synthesis of benzene from acetylene on $\operatorname{Pd}(111)$. It has already been demonstrated that palladium has an almost unique activity for acetylene tricyclization as compared with other transition metals, giving an efficiency of about $\simeq 30 \%$ of the acetylene converted to benzene $[9,10,11,12]$. In addition, this reaction is clearly affected by the

ๆ To whom any correspondence should be addressed. E-mail: jlr@linux0.unsl.edu.ar. 
surface structure; the efficiency of the reaction decreases by a factor of 20 from the (111) to the (100) face and no reaction is found on the (110) surface [12,13]. The threefold symmetry in the (111) face of the catalyst correctly orients the adsorbed acetylene to facilitate benzene synthesis.

This paper addresses theoretical strategies for analytical estimation of reaction yields by reactive ensembles chemisorbed on regular surfaces, assuming that the adsorption of the reactant proceeds at random. A comparison between analytically calculated, simulated and experimental reaction yields is carried out for acetylene conversion to benzene on $\operatorname{Pd}(111)$.

\section{The physical model}

The physical system is defined as molecules of a reactant adsorbed on a surface having a structure of adsorption sites. It is assumed that the reactant molecule occupies in general several adsorption sites. The equilibrium state of the adsorbed phase is characterized by the surface coverage $\theta$ (defined as the fraction of occupied adsorption sites). On the other hand the product molecule requires an $n$-reactant ensemble arranged in the correct geometry $T$ (hereafter we call any set of $n$-reactants with the correct geometry $T$ a 'template'). The probability of an arbitrary molecule being one forming a template $T$ can be symbolically expressed as $P_{T}$. Wherever a proper configuration $T$ occurs on the surface the $n$-ensemble reacts forming a product molecule which desorbs from the surface. The reaction yield $Y(\theta)$ is defined as $Y(\theta)=N_{r}(\theta) / N\left(\theta_{j}\right)$, where $N_{r}(\theta)$ is the number of reactant molecules leaving the surface as reaction product (for a given initial $\theta$ ) and $N\left(\theta_{j}\right)$ is the total number of adsorbed molecules at the maximum attainable coverage on the surface, $\theta_{j} . Y(\theta)$ can be rewritten using probability language as

$$
Y(\theta)=\frac{N(\theta) P_{r}(\theta)}{N\left(\theta_{j}\right)}
$$

where $P_{r}(\theta)$ denotes the probability of an arbitrary molecule belonging to a product molecule. There are in general $T_{1} \ldots T_{k}$ distinguishable configurations of the basic template $T$ having different orientations. Since the reactant molecules can be shared between these templates, the probability $P_{r}$ is not merely given by $P_{T}$. The simplest case is that when two templates sharing a molecule are isolated; they can give rise to only one product molecule. It is easier to estimate $P_{r}$ through the complementary event. So, $P_{T}=P_{T}\left(P_{r \mid T}+P_{s \mid T}\right)=P_{r}+P_{s}$, where $P_{s}$ is the probability of a molecule being initially in a template $T$ and even so remaining on the surface after the reaction step. Accordingly, $P_{r}=P_{T}-P_{s}$. The term $P_{s}$ has in general a very complicated form provided that for a molecule forming a template, the chance of staying on the surface depends on how this template is sharing molecules with neighbouring similar templates and how the later ones are connected to others, and so on. Strictly speaking, It depends on the configuration of the set of connected templates in which the particle is embedded. However, it can be explicitly written if the whole set of template configurations that can be obtained by combining the basic $T_{1} \ldots T_{k}$ sharing their limits is split into mutually exclusive subsets $S_{i}$ containing those configurations having exactly $i$ templates from the set $T_{1} \ldots T_{k}$. Accordingly

$$
P_{r}=P_{T}-\left(\sum_{i=2}^{\infty} K_{i} P_{S_{i}}\right)=P_{T}-\sum_{i=2}^{\infty}\left(K_{i}-K_{i-1}\right) P_{S_{i}}^{c} .
$$

$P_{S_{i}}\left(P_{S_{i}}^{c}\right)$ is the probability of finding an arbitrary molecule in a template configuration $S_{i}$ ( $S_{i}$ or larger), and $K_{i}$ is the probability of it remaining in the surface given that it is already part of $S_{i}\left(K_{1}=0\right.$ because any isolated $T$ reacts). The terms $P_{S_{i}}^{c}$ are powers of 
the surface coverage increasing with the index $i$ and only a few terms of the summation make an appreciable contribution to $P_{r}$. We illustrate how $Y(\theta)$ is calculated in the case of benzene synthesis from acetylene on $\operatorname{Pd}(111)$, based upon equations (1) and (2).

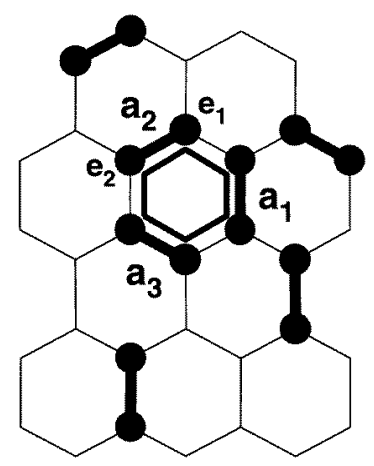

Figure 1. The proposed scheme of acetylene molecules (dimers) adsorbed on a hexagonal lattice. A reactive template $T$ giving rise to a benzene molecule is shown (the hexagonal ring). The three possible orientations of dimers are denoted $a_{1}, a_{2}, a_{3}$. The two sites referred to in the text as $e_{1}$ and $e_{2}$ are also shown.

\section{Application to benzene synthesis from acetylene on $\operatorname{Pd}(111)$}

Much evidence exists about the proper configurations of neighbouring acetylene molecules to give rise to tricyclization [14]. The acetylene configuration has been calculated to be a ring around a hollow site [15]. The relative positions of the acetylene molecules can be modelled assuming that they adsorb as dimers on a hexagonal lattice occupying two adjacent sites. The jamming coverage, i.e. the maximum coverage that can be obtained by randomly filling the lattice with dimers, has already been calculated by many methods and it is known to be $\theta_{j}=0.44$ [1], compared with an experimental value of $\theta_{j}=0.46$ $[9,12]$. A benzene ring will be formed around the hexagon if three molecules can adsorb forming a hexagonal ring (figure 1). It is tacitly assumed that the reaction probability for a hexagonal ring 'a priori' equals one. When two or more reactive hexagons are sharing reactant molecules their effective reaction probability is statistically diminished, as stated in deriving equation (2). However, the only constraint for the reaction to be chemically feasible is to have three dimers (acetylene) geometrically arranged in a hexagonal template (see figure 1), independently of the state of the neighbouring sites. Hence the reaction path of the reactive template is independent of the surface coverage and only the effective probability of reacting and forming benzene is statistically coverage dependent. This is the simplest assumption consistent with the theoretical evidence available on the relevant system [12, 15].

It is known that the reaction proceeds through an intermediate step by which acetylene first dimerizes forming $\mathrm{C}_{4} \mathrm{H}_{4}$, and then reaction with further acetylene gives benzene $[16,17]$. However, details of the reaction path are ignored in this model which rather relies on the initial and final states of the reactive ensemble. There are two distinguishable configurations of the basic template $T$, denoted $T_{1}, T_{2}$ (see figure 2 ). Since there are three equally probable orientations for the acetylene molecule on the hexagonal lattice, say, $a_{i}, i=1,2,3$, we use the notation $a_{i j}, i=1,2,3, j=1,2$, for the molecule with 

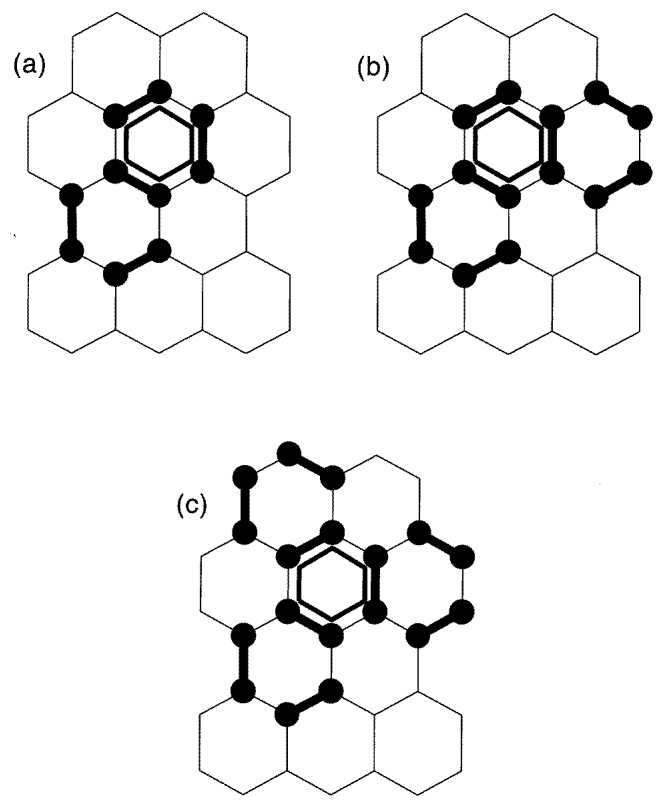

Figure 2. Given a ring, filled circles, each part of the ring can be forming part of an extra ring. The simplest configurations $S_{i}$ are shown in: (a) configuration $S_{2}$ (two templates sharing a molecule), $K_{2}=2 / 5$; (b) $S_{3}$, (three templates sharing two molecules), $K_{3}=4 / 7$; (c) $S_{4}$ (four templates sharing three molecules), $K_{4}=2 / 3$.

orientation $i$ in the template $T_{j}$. According to this, the events $T_{j}$ and $T$ are expressed as $T_{j}=a_{1 j} \cup a_{2 j} \cup a_{3 j}$ and $T=T_{1} \cup T_{2}=\left(a_{11} \cup a_{21} \cup a_{31}\right) \cup\left(a_{12} \cup a_{22} \cup a_{32}\right)$. Provided that $a_{i j} \cap a_{i^{\prime} j^{\prime}}=\emptyset \forall i \neq i^{\prime}, j, j^{\prime}$, by definition, $P_{T}=P_{T_{1} \cup T_{2}}=P_{T_{1}}+P_{T_{2}}-P_{T_{1} \cap T_{2}}$ finally yields

$$
\begin{aligned}
P_{T}=P_{a_{11} \cup a_{21} \cup a_{31}}+P_{a_{12} \cup a_{22} \cup a_{32}}-P_{\left(a_{11} \cap a_{12}\right) \cup\left(a_{21} \cap a_{22}\right) \cup\left(a_{31} \cap a_{32}\right)} \\
P_{T}=P_{a_{11} \cup a_{21} \cup a_{31}}+P_{a_{12} \cup a_{22} \cup a_{32}}-\left(P_{a_{11} \cap a_{12}}+P_{a_{21} \cap a_{22}}+P_{a_{31} \cap a_{32}}\right. \\
-P_{\left(a_{11} \cap a_{12}\right) \cap\left(a_{21} \cap a_{22}\right)}-P_{\left(a_{11} \cap a_{12}\right) \cap\left(a_{31} \cap a_{32}\right)}-P_{\left(a_{21} \cap a_{22}\right) \cap\left(a_{31} \cap a_{32}\right)} \\
\left.+P_{\left(a_{11} \cap a_{12}\right) \cap\left(a_{21} \cap a_{22}\right) \cap\left(a_{31} \cap a_{32}\right)}\right)
\end{aligned}
$$

where the cross-term probabilities appear because dimers can be shared between two templates. The terms $a_{i j}$ account for the probability of the molecules belonging to at least one template ( $S_{1}$ or larger), and the cross-terms account for the probability of being shared between two ( $S_{2}$ or larger), three ( $S_{3}$ or larger) and four ( $S_{4}$ or larger) templates. One example of each of these configurations is shown in figure $2-$ (a): $S_{2} ;$ (b): $S_{3}$; and (c): $S_{4}$. Using equation (2)

$$
\begin{aligned}
& P_{r}=P_{S_{1}}^{c}-\left(P_{S_{2}}^{c}-P_{S_{3}}^{c}+P_{S_{4}}^{c}\right)-\left(K_{2} P_{S_{2}}^{c}+\left(K_{3}-K_{2}\right) P_{S_{3}}^{c}+\left(K_{4}-K_{3}\right) P_{S_{4}}^{c}+\ldots\right) \\
& P_{r}=P_{S_{1}}^{c}-\left(1+K_{2}\right) P_{S_{2}}^{c}+\left(1-\left(K_{3}-K_{2}\right)\right) P_{S_{3}}^{c}-\left(1-\left(K_{4}-K_{3}\right)\right) P_{S_{4}}^{c} .
\end{aligned}
$$

If the number of molecules necessary to build up the species $S_{i}$ is $N_{i}$ then $P_{S_{i}}^{c}$ can be written as

$$
P_{S_{i}}^{c} \propto P_{2 \mathrm{nd} \mid 1 \mathrm{st}} P_{3 \mathrm{rd} \mid \text { st } 2 \mathrm{nd}} \ldots P_{N_{i} \mathrm{th} \mid 1 \mathrm{st} \ldots\left(N_{i}-1\right) \text { th }}
$$

where $P_{\left.N_{i} \text { th } \mid 1 \text { st...(Ni } N_{i}-1\right) \text { th }}$ is the probability of having the $N_{i}$ th molecule in the correct orientation to build the $S_{i}$-species, given that $N_{i}-1$ molecules are already in the correct 
configuration. In our case, $N_{1}=3, N_{2}=5$ and $N_{4}=9$. The proportionality constant equals the number of distinguishable configurations of $S_{i}$ provided that the direction of the first molecule is given.

We show, as an example, how $S_{1}$ is calculated in this application. $S_{1}$ requires three molecules to be in a ring configuration. As stated above, it is only assumed that the molecules are adsorbed at random with all possible orientations equally probable. Given an arbitrary molecule $a_{1}$ (see figure 1 ) adsorbed on the surface, the second dimer can be accommodated by first placing one of its atoms on the site $e_{1}$ (with probability $2 \theta$ ) and then choosing the right orientation (with probability $1 / 2$ ); so $P_{2 \text { nd } \mid 1 \text { st }}=\theta$. However, with the only condition that the first dimer is in the given position, the same can be accomplished by starting at the site $e_{2}$, giving $P_{2 \text { nd| } 1 \text { st }}=\frac{2}{3} \theta$. Provided that these two ways are $a$ priori equally probable, $P_{2 \text { nd } \mid 1 s t}=\left(\theta+\frac{2}{3} \theta\right) / 2=\frac{5}{6} \theta$. Following the same argument we obtain $P_{3 \mathrm{rd} \mid \mathrm{st} 2 \mathrm{nd}}=\theta$. Thus, $P_{S_{1}}^{c}=2 \times \frac{5}{6} \theta^{2}$. For the configurations $S_{2}$ and $S_{3}$ we obtain $P_{S_{2}}^{c}=\left(\left(\frac{5}{6}\right)^{2} \theta^{4}\right)$ and $P_{S_{3}}^{c}=2 \times\left(\left(\frac{5}{6}\right)^{3} \theta^{6}\right)$. The values of $K_{i}$ are obtained assuming that the most-connected template $T$ in every configuration reacts (see figure 2); so $K_{2}=2 / 5$ (two out of five dimers remain on the surface), $K_{3}=4 / 7$ and $K_{4}=6 / 9$. (This is mentioned here only for the sake of consistency, because in this system only $S_{1}$ and $S_{2}$ make an appreciable contribution to the reaction yield and this assumption is no longer necessary.) Finally, on replacing the calculated probabilities in equation (4), and using the fact that $N(\theta) \propto \theta$, the reaction yield $Y(\theta)$ gives

$$
Y(\theta)=\frac{\theta^{3}}{\theta_{j}}\left(\frac{5}{3}-\frac{35}{36} \theta^{2}+\frac{725}{756} \theta^{4}-\cdots\right) .
$$

The higher-order contributions were omitted because the last term included in equation (5) is already so small as to contribute only $2 \%$ of the total reaction yield at maximum coverage.

This result gives a maximum of $29.5 \%$ of acetylene converted to benzene at saturation coverage $\theta_{j}=0.44$ which is in very good agreement with the value obtained from experimental measurements of the reaction in UHV that gives $30 \pm 2 \%[9,14]$. This agreement is also qualitatively extended over the range of surface coverage (figure 3 ).

A Monte Carlo simulation of random deposition of dimers and subsequent reaction on a hexagonal lattice was also performed. The simulation was carried out on a lattice of $10^{6}$ sites. In order to calculate reaction yield at a given surface coverage we followed two main steps as detailed below.

(1) The RSA of dimers proceeded until the prespecified coverage $\theta$ was obtained.

(2) The reaction step was carried out. All possible reactive templates (hexagonal rings) on the surface were tagged and the reaction product was obtained by randomly picking up a ring from the set of tagged rings, desorbing it from the surface and counting three dimers as part of the reaction yield $Y(\theta)$. This step was repeated until the rings were exhausted. The list of tagged rings was updated after each single step because the desorbed dimer could also be part of another reactive ring (see the central ring in figure 2(b)). Finally, the reaction yield at the given initial coverage was calculated as the total number of dimers desorbed in the reaction step divided by the number of dimers adsorbed at the jamming coverage.

It is worth mentioning that the reactive phase consists mostly of single rings and double rings (like those shown in figures 1 and 2(a)). More complex structures of connected rings (see figure 2(b)) are sparsely distributed over the surface. They contribute only a few per cent $(2-3 \%)$ to the reaction yield at the jamming coverage. Moreover, ordered structures like the one shown in figure 2(c) (or larger) are rare events in the adsorbed phase. Even though 


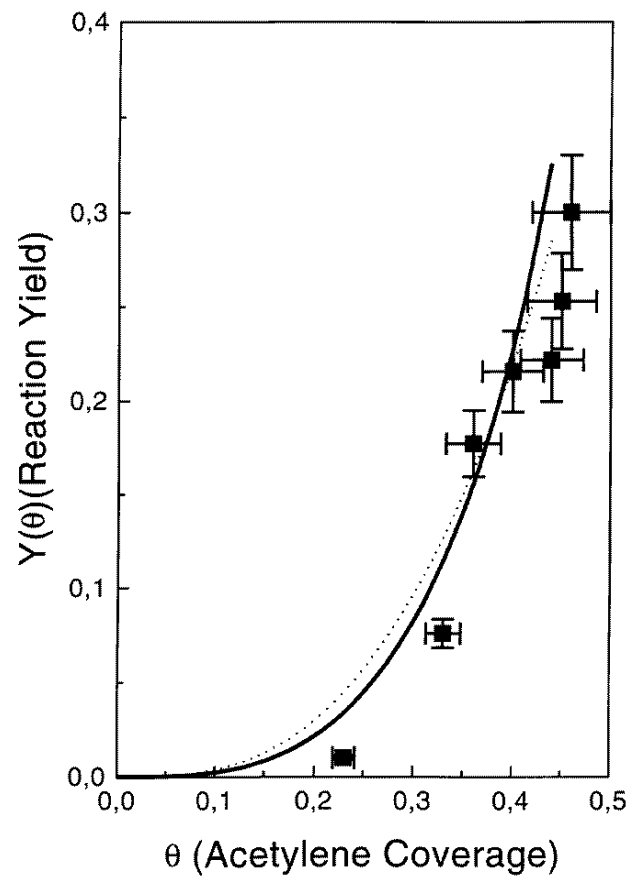

Figure 3. A plot of the reaction yield $Y(\theta)$ following acetylene adsorption on $\operatorname{Pd}(111)$. Filled circles with error bars correspond to experimental data from [1,11]. The solid and dashed lines show the simulation and analytical results obtained using equation (6), respectively.

at high coverages $\left(\theta>\theta_{c} / 2=0.326\right.$ where $\theta_{c}=0.6527$ is the percolation threshold of bond percolation for the honeycomb lattice) adsorbed dimers are highly connected throughout the surface (as defined in simple percolation theory [18]) with long-ranged spatial correlations, the reactive templates are sparsely embedded in the adsorbed phase, so they can be considered as independent ordered local structures having no long-ranged correlations. This fact partially supports the analytical approximation based upon a local analysis of the reaction probabilities. In addition, the comparison between simulation (which may be considered as the exact result within statistical error) and analytical calculations shows that the assumption works well at all surface coverages. Ultimately, the long-ranged correlations of dimers at coverages higher than the percolation threshold $\theta \approx 0.33$ seem to have no major effect on the statistics of benzene templates which remain short-range correlated even at high coverages.

\section{Conclusions}

Figure 3 shows a comparison between the simulated and analytical results from equation (5) along with the experimental benzene yield from [9]. Model as well as simulation calculations are in qualitative agreement with the experimental findings of Tysoe et al, not only as regards the maximum amount of benzene predicted to be formed on the $\mathrm{Pd}(111)$ surface but also as regards the behaviour of the benzene yield as a function of acetylene coverage. In particular, the maximum reaction yield predicted by using the model from equation (5) (namely 29.5\%) is in excellent agreement with the well established experimental value 30\% [9]. This is the 
first calculation and MC simulation accounting for that value in this system.

These results have been obtained with the one assumption that the acetylene is chemisorbed at random with all its possible orientations on the lattice equally probable, and no fitting parameters have been introduced in the physical model. The comparison with experimental data also supports the idea that the reaction efficiency may be mainly controlled by configurations of adsorbed acetylene molecules on a regular lattice of chemisorption sites under diffusionless RSA. This gives an example of the constraints introduced by the geometry of the surface that affects the efficiency of a complex reaction. At low surface coverage $(\theta<0.33)$ the simulation comes closer than the analytical study to the experimental behaviour; however, the experimental reaction yield is still lower than that predicted by the model. One reason for the difference might be that the reaction probability for the reactive ring of acetylenes at low coverage is less than unity (as we assumed in the present calculation and simulation). Nevertheless, to the best knowledge of the authors there is no experimental 'clue' regarding this point other than a vanishing reaction yield measured for $\theta<0.2$ [9]. Another perhaps more plausible reason for the observed difference can be attributed to the existence of lateral interactions between neighbouring acetylenes which has not been considered in the present model. It is known that acetylene can adsorb on $\operatorname{Pd}(111)$ presenting a molecular plane tilted away from the surface normal $[9,14,15,19,20]$. This may give rise to repulsive interactions between nearest-neighbour acetylenes and consequently decrease the reaction yield. Additional effects such as mobility or reordering of acetylene after chemisorption were not taken into account in the present study (which relies on a diffusionless RSA). However, the agreement between the jamming coverage $\theta_{j}=0.44$ for RSA of dimers on a hexagonal lattice and the experimental value 0.46 suggests that the spatial arrangement of acetylene on the surface may be fairly represented by an RSA process as assumed here.

The chemistry of the acetylene conversion to benzene on palladium is determined by the chemical properties of the surface, but the efficiency of the reaction appears to be determined by the stochastic geometry of the acetylene adsorbed phase on the regular lattice of chemisorption sites.

\section{Acknowledgment}

One of the authors (JLR) wishes to thank Professor W Steele for the hospitality of his research group at 152 Davey Laboratory, Department of Chemistry, The Pennsylvania State University. JLR is on leave with a CONICET fellowship (Argentina) at the above address.

\section{References}

[1] For a comprehensive review and reference list, see Evans J W 1994 Rev. Mod. Phys. 651281

[2] Roberts J K 1935 Nature 1351037

[3] Flory P J 1939 J. Am. Chem. Soc. 611518

[4] Brundle C R, Behm R J and Barker J A 1984 J. Vac. Sci. Technol. A 21038

[5] Chang S L and Thiel P A 1987 Phys. Rev. Lett. 59296

[6] Evans J W 1987 J. Chem. Phys. 873038

[7] Ziff R M, Gulari E and Barshad Y 1986 Phys. Rev. Lett. 562553

[8] Dumont M, Dufour P, Sente B and Daggonier R 1990 J. Catal. 12295

Evans J W and Miesh M S 1991 Phys. Rev. Lett. 66833

Kohler J and ben-Avraham D 1991 J. Phys. A: Math. Gen. 24 L621

Nord R S and Evans J W 1985 J. Chem. Phys. 822795

Maltz A and Albano E V 1992 Surf. Sci. 277414 
[9] Tysoe W T, Nyberg G L and Lambert R M 1983 Surf. Sci. 135128

[10] Sesselman W, Woratchek B, Ertl G, Kuppers J and Haberland H 1983 Surf. Sci. 130245

[11] Gentle T M and Muetterties E L 1983 J. Phys. Chem. 87245

[12] Lambert R M and Ormerod R M 1994 Surface Reactions (Springer Series in Surface Sciences 34) ed R J Madix (Berlin: Springer)

[13] Yoshinobu J, Sekitani T, Onchi M and Nishijima M, 1990 J. Phys. Chem. 944269

[14] Hoffmann H, Zaera F, Ormerod R M, Lambert R M, Yao Y M, Saldin D K, Wang D P, Bennett D W and Tysoe W T, 1992 Surf. Sci. 2681

[15] Pacchionni G and Lambert R M 1994 Surf. Sci. 304208

[16] Ormerod R M, Lambert R M, Hoffmann H, Zaera F, Wang L P, Bennett D W and Tysoe W T 1993 Surf. Sci. 277277

[17] Hoffmann H, Zaera F, Ormerod R M, Lambert R M, Yao J M, Saldin D K, Wang L P, Bennett D W and Tysoe W T 1992 Surf. Sci. 2681

[18] Stauffer D 1985 Introduction to Percolation Theory (London: Taylor \& Francis)

[19] Gates J A and Kesmodel L L 1983 Surf. Sci. 12468

[20] Sellers H 1990 J. Phys. Chem. 948329 